\title{
É possível viver de day-trade em ações?
}

\author{
(Day-trading stocks for a living?)
}

\section{Fernando Chague ${ }^{\dagger}$ \\ Bruno Giovannetti ${ }^{+}$}

\begin{abstract}
Resumo O número de pessoas que tem tentado usar o day-trade como fonte de renda regular vem aumentando. Nesta nota analisamos os resultados de day-trade em ações. Partindo de todos os 98.378 indivíduos que começaram a fazer day-trade em ações no Brasil entre 2013 e 2016, e operaram até 2018, encontramos que apenas 127 indivíduos foram capazes de apresentar lucro bruto diário médio acima de 100 reais por mais de 300 pregões.
\end{abstract}

Palavras-chave: Day trade; Viver de day trade; Investidores individuais.

Código JEL: G41, G50, D14.

\begin{abstract}
The number of individuals trying to day-trade for a living has been increasing worldwide. In this note, we study the performance of all 98,378 individuals who started to day-trade stocks in Brazil between 2013 and 2016. After analyzing all their daytrades in stocks from 2013 to 2018 , we find that only 127 individuals presented an average daily gross profit higher than $\mathrm{R} \$ 100$ for more than 300 days.
\end{abstract}

Keywords: Day trade; Day trading for a living; Retail investors.

JEL Code: G41, G50, D14.

\section{Introdução}

Day-trading é a atividade de comprar e vender o mesmo ativo financeiro, no mesmo dia e na mesma quantidade. $\mathrm{O}$ day-trader lucra quando seu preço médio de venda é maior do que seu preço médio de compra, descontando-se os custos de operação.

O número de pessoas que buscam no day-trade uma fonte regular de renda vem aumentando no Brasil e no mundo. ${ }^{1}$ No entanto, são poucas as estatísticas claras sobre o real desempenho de quem tenta seguir por esse caminho.

Submitted on July 29, 2020. Revised on July 30, 2020. Accepted on August 1st, 2020. Published online in September 2020. Editor in charge: Alan de Genaro.

${ }^{\dagger}$ Sao Paulo School of Economics - FGV, Brazil: fernando. chague f gv . br

¥Sao Paulo School of Economics - FGV, Brazil: bruno.giovannetti@ fgv . br

${ }^{1}$ Reportagens na mídia internacional e nacional sobre o assunto: 1 . https ://www. ft.com/con tent/45d0a047-360f-4abf-86ee-108f436015a1; 2. https://www. forbes.com/sites /simonmoore/2020/06/18/day-trading-surges-in-popularity-this-wont-end-we 11/\#2565070b56c8; 3. https://markets.businessinsider.com/news/stocks/warr en-buffett-howard-marks-will-ultimately-beat-day-traders-malkiel-20206-1029321160; 4. https://blog.wealthfront.com/the-day-trading-pandemic/; 5. https://www.cnnbrasil.com.br/business/2020/04/01/day-trade-o-que-e-comofazer-e-vale-a-pena; 6. https://exame.com/mercados/day-trade-e-cassino-mui to-mais-sorte-do-que-tecnica-diz-pesquisador/. 
Chague et al. (2020) apresentam essas estatísticas para as pessoas que começam fazer day-trade no mercado brasileiro de contratos futuros (contratos de mini-índice). Os autores analisam todos os indivíduos que começaram a fazer day-trade nesse mercado entre 2013 e 2015 (19.646 indivíduos) e que persistiram por mais de 300 pregões (1.551 indivíduos). Entre os 1.551 que persistiram, apenas 8 conseguiram apresentar lucro bruto diário médio maior do que a remuneração de entrada de um caixa de banco (160 reais por dia). Além desse estudo, Barber et al. (2014) e Barber et al. (2019) apresentam resultados qualitativamente parecidos para a performance de day-traders persistentes em mercados estrangeiros.

Nesta nota aplicamos a mesma metodologia de Chague et al. (2020) focando no day-trade no mercado à vista de ações brasileiro. Procuramos assim complementar o conjunto de informação dos aspirantes a day-trader. Os resultados obtidos também não são animadores.

\section{Resultados}

O banco de dados vem da Comissão de Valores Mobiliários (CVM). O banco contém a atividade de trading completa de todos os indivíduos em todas as ações em todos os dias de 2012 a 2018. As observações estão no nível indivíduo-dia-ação. ${ }^{2}$ Para cada indivíduo-dia-ação, observamos a quantidade comprada, a quantidade vendida, o volume comprado e o volume vendido. Dizemos que um indivíduo realizou uma operação de day-trade em um determinado dia-ação, quando a quantidade comprada é igual à quantidade vendida. Indivíduos são identificados por um número-fantasia anônimo, garantindo assim o sigilo pessoal das informações.

Nosso objetivo é entender se é possível ganhar dinheiro com day-trade de maneira regular e se há aprendizado na atividade. Para isso, acompanhamos cada day-trader desde seu primeiro dia. Utilizamos o ano de 2012 como base para definir os iniciantes: se um indivíduo não aparece com nenhum day-trade em 2012, consideramos que ele inicia a atividade de day-trade no momento de sua primeira aparição nos anos à frente (todos os indivíduos que fizeram algum day-trade em 2012, 51.182 indivíduos, são descartados da amostra, pois não sabemos quando eles começaram). Além disso, para termos amostra suficiente para estudarmos pelo menos 300 pregões de cada indivíduo, também excluímos da análise todos os indivíduos que fizeram seu primeiro day-trade após 2017 (102.595 indivíduos). Em suma, focamos este estudo em todos os indivíduos que começaram a fazer day-trade em ações no

${ }^{2}$ Os dados utilizados neste estudo foram obtidos sob o convênio de pesquisa NEFIN-USP/5988 (processo CVM 19957.003353/2015-85). 
Brasil entre 2013 e 2016, um total de 98.378 indivíduos, e em todas as suas operações de day-trade entre 2013 e 2018.

Dos 98.378 indivíduos que fizeram seu primeiro day-trade em ações no período entre 2013 e 2016, apenas 554 fizeram day-trades em mais de 300 pregões. Todos os outros indivíduos $(99,43 \%)$ não persistiram na atividade (apresentam menos de 300 pregões com day-trades em ações). Essa é a primeira informação relevante para alguém que esteja interessado em viver de day-trade: a taxa de desistência parece ser extremamente alta.

Como foi a performance dos 554 day-traders que persistiram por mais de 300 pregões? Para respondermos essa pergunta, calculamos primeiramente o "lucro bruto diário" de um indivíduo, descontando do resultado de cada dia de day-trade $0,025 \%$ do volume total das operações de day-trade, a título de custos de emolumentos e taxa de liquidação, e 50 reais fixos por dia (independentemente do número de empresas negociadas), a título de "outros custos" (por exemplo, possíveis gastos com corretagem, plataformas de trading, cursos, livros, computadores, internet, etc). É importante enfatizar que não descontamos os possíveis gastos com imposto de renda. Calculamos então o "lucro bruto diário médio" para cada um dos 554 indivíduos que persistiram por mais de 300 pregões: a média dos lucros brutos diários de cada indivíduo.

A média do "lucro bruto diário médio" entre os 554 indivíduos é 49 reais negativos e a mediana 62 reais negativos. Além disso, apenas 127 indivíduos apresentaram "lucro bruto diário médio" acima de 100 reais. Acima de 300 reais diários, temos apenas 76 indivíduos.

Ou seja, partindo de todos os indivíduos que começaram a fazer daytrade em ações no mercado brasileiro entre 2013 e 2016 (98.378 indivíduos), temos:

(i) 99,43\% dos indivíduos não persistiram na atividade (apresentaram menos de 300 pregões com day-trades);

(ii) considerando-se os day-traders que persistiram (operaram por mais de 300 pregões), a performance média foi negativa;

(iii) apenas 127 indivíduos foram capazes de apresentar lucro bruto diário médio acima de 100 reais em mais de 300 pregões.

É possível que haja um período de aprendizado que deva ser descontado ao calcularmos essas estatísticas. No entanto, tal cuidado não altera a conclusão; ou seja, não parece haver aprendizado. Se calcularmos o lucro bruto diário médio de cada um dos 554 indivíduos excluindo os 200 primeiros pregões de cada um (um possível período de aprendizado), os resultados não se 
alteram significativamente. A média entre os 554 indivíduos de seus lucros brutos diários médios vai para 91 reais negativos (ou seja, até piora) e a mediana continua em 62 reais negativos; 130 indivíduos, ao invés de 127, passam a apresentar lucro bruto diário médio acima de 100 reais.

Por fim, avaliamos brevemente a performance dos 127 indivíduos que apresentaram lucro bruto diário médio acima de 100 reais. Entre esses 127 indivíduos, temos os seguintes lucros brutos diários médios: 103 reais, para o indivíduo com o menor lucro bruto diário médio (o desvio-padrão do lucro bruto diário desse indivíduo foi de 747 reais); 370 reais, para o indivíduo mediano (o desvio-padrão do lucro bruto diário desse indivíduo foi de 2.286 reais); 4.032 reais, para o indivíduo com o maior lucro bruto diário médio (o desvio-padrão do lucro bruto diário desse indivíduo foi de 33.888 reais). Ou seja, mesmo considerando apenas os 127 indivíduos "ganhadores", vemos uma média de ganho baixa frente ao risco.

\section{Agradecimentos}

Agradecemos aos editores Marcelo Fernandes e Alan De Genaro pelos comentários.

\section{Referências}

Barber, B. M., Lee, Y.-T., Liu, Y.-J. e Odean, T. (2014). The cross-section of speculator skill: Evidence from day trading, Journal of Financial Markets 18: $1-24$.

URL: http: / /www.sciencedirect.com/science/article/ pii/s1386418113000190

Barber, B. M., Lee, Y.-T., Liu, Y.-J., Odean, T. e Zhang, K. (2019). Learning, Fast or Slow, The Review of Asset Pricing Studies 10(1): 61-93.

URL: https://doi .org/10.1093/rapstu/raz006

Chague, F., De-Losso, R. e Giovannetti, B. (2020). Day trading for a living? Available at SSRN: https://ssrn.com/abstract $=3423101$. 